\title{
Magnetic resonance characteristics of adult-onset Lhermitte-Duclos disease: An indicator for active cancer surveillance?
}

\author{
GUANGQUAN WEI $^{1}$, WEI ZHANG ${ }^{2}$, ${\text { QINLONG } \text { LI }^{3}, \text { XIAOWEI KANG }}^{1}$, HAITAO ZHAO $^{1}$, \\ XIANPING LIU ${ }^{1}$, XING TANG ${ }^{1}$, YUANMING WU ${ }^{4}$, JUNTAO HAN ${ }^{5}$ and HONG YIN ${ }^{1}$
}

${ }^{1}$ Molecular Imaging Center, Department of Radiology, Xijing Hospital, The Fourth Military Medical University, Xi'an, Shaanxi 710032; ${ }^{2}$ Department of Cardiology, Tangdu Hospital, The Fourth Military Medical University, Xi'an, Shaanxi 710038; ${ }^{3}$ Department of Pathology, Xijing Hospital; ${ }^{4}$ Center for Gene Typing; ${ }^{5}$ Department of Surgery, Xijing Hospital, The Fourth Military Medical University, Xi'an, Shaanxi 710032, P.R. China

Received August 28, 2013; Accepted January 14, 2014

DOI: $10.3892 / \mathrm{mco} .2014 .258$

\begin{abstract}
Lhermitte-Duclos disease (LDD) is a rare, non-cancerous entity characterized by enlarged, abnormally developed cerebellar folia containing dysplastic cells. Symptomatic LDD is commonly observed in adults (adult-onset LDD, aLDD) as an isolated condition or associated with Cowden's disease (CD). The present study aimed to investigate the magnetic resonance imaging (MRI) characteristics and the underlying pathological findings in 7 cases of aLDD, with emphasis on the association with $\mathrm{CD}$ and the need for active cancer surveillance once the diagnosis of LDD is confirmed. The MRI findings along with the clinical and histopathological data collected from 7 patients with aLDD were retrospectively reviewed. The diagnosis of $\mathrm{CD}$ was based on a range of clinical characteristics, according to the International Cowden Consortium Criteria. A thorough review of the published data was conducted and our results indicated that all 7 cases shared similar MRI characteristics, whether the aLDD was sporadic ( 2 cases) or associated with CD (5 cases), including a highly typical non-enhancing striated MRI appearance of thickened folia, consisting of alternating bands on T1- and T2-weighted
\end{abstract}

Correspondence to: Professor Hong Yin, Molecular Imaging Center, Department of Radiology, Xijing Hospital, The Fourth Military Medical University, 127 Changle West Road, Xi'an, Shaanxi 710032, P.R. China

E-mail: yinhong@fmmu.edu.cn

Professor Juntao Han, Department of Surgery, Xijing Hospital, The Fourth Military Medical University, 127 Changle West Road, Xi'an, Shaanxi 710032, P.R. China

E-mail: radiologic@163.com

Key words: Lhermitte-Duclos disease, dysplastic cerebellar gangliocytoma, Cowden's disease, magnetic resonance imaging, pathology images. On gross examination, the involved cerebellar folia were distorted and enlarged, whereas the histopathological examination revealed that the molecular layer was widened and occupied by abnormal ganglion cells. Moreover, a reduction in the number or absence of the Purkinje cells and hypertrophy of the granular cell layer were observed. Our findings were consistent with the diagnosis of LDD. Variable levels of vacuolization of the white matter and the molecular layer were observed in all the cases. Notably, CD34 immunohistochemical analysis revealed the presence of angiogenesis within the lesions. aLDD associated with CD exhibited no pathological or immunohistochemical characteristics that were distinct from those of isolated aLDD. Of the 7 cases of aLDD, 5 presented with symptoms suggestive of $C D$, which is a syndrome associated with a high risk of multiple benign and malignant neoplasms. In conclusion, aLDD exhibits characteristic MRI and histopathological findings and displays a strong association with CD. Therefore, we recommend that the MRI diagnosis of aLDD triggers active cancer surveillance and preventive care.

\section{Introduction}

Lhermitte-Duclos disease (LDD), also referred to as dysplastic cerebellar gangliocytoma, is a rare, but clinically significant type of cerebellar disorder that corresponds histologically to a hamartoma. Lhermitte and Duclos (1) were the first to describe this cerebellar condition in 1920. LDD is a slowly enlarging, non-cancerous entity, characterized by enlarged, abnormally developed cerebellar folia, containing dysplastic rather than neoplastic cells. Symptomatic LDD may occur at any age, but most commonly affects adults (adult-onset LDD, aLDD), as an isolated condition or associated with Cowden's disease (CD). Accumulating evidence indicates that aLDD is pathognomonic for CD (2-5), which is a multiple hamartoma syndrome with an increased risk for benign and malignant tumors of the thyroid gland, breast, endometrium and other organs (5-7). To the best of our knowledge, 250 LDD cases have been reported in the medical literature to date, 53 of which were associated with CD (3,8-10). 
Magnetic resonance imaging (MRI) is considered to be the imaging modality of choice for the diagnosis of LDD and an Aunt Minnie diagnosis of LDD may be confirmed due to the characteristic 'tiger-striped' appearance of LDD on MRI (11-14). An improved understanding of the underlying molecular pathogenesis and affected signaling pathways of aLDD and its association with CD may have direct implications for optimizing current treatments and developing novel therapeutic approaches.

This study aimed to investigate the MRI characteristics and underlying histopathological findings in 7 cases of aLDD, with emphasis on the association between aLDD and CD and the characteristic MRI appearance as an imaging marker for active cancer surveillance and preventive care of this rare condition.

\section{Materials and methods}

Patients and data. The medical records of 7 patients diagnosed with LDD between January, 1999 and December, 2011 were retrieved from our hospital database in accordance with human subject research protocols and were retrospectively reviewed. All the patients had been treated with surgical resection and the diagnosis was histopathologically confirmed. All the patients underwent complete examination, including thyroid and breast ultrasonography, in order to collect clinical evidence suggestive of associated CD. A clinical diagnosis of $\mathrm{CD}$ was made when an individual met the operational criteria established by the International Cowden Consortium (6). The PubMed database was searched for previous cases of LDD, particularly those associated with $\mathrm{CD}$, using the key terms 'Lhermitte-Duclos disease' and 'Cowden's disease'.

Our retrospective study was approved by the Institutional Ethics Review Board of the Fourth Military Medical University (Xi'an, China) and all the patients provided informed consent to the use of their clinical and imaging data for research purposes.

MRI protocol and analysis. All 7 patients underwent MRI in our hospital, which was performed with different scanners: two with a Gyroscan Intera Master 1.5T MR system (Philips Medical Systems, Best, The Netherlands) and five 3-Tesla Siemens Trio MRI scanners (Siemens Medical Solutions, Erlangen, Germany). Images were obtained in the transverse plane with a T2-weighted turbo-spin-echo sequence of 4,774/110 (repetition time/echo time), a T1-weighted spin-echo sequence of 476/15 and a fluid-attenuated inversion-recovery (FLAIR) turbo-spin-echo sequence of 6,000/100/2,000 (repetition time/echo time/inversion time). Images were also obtained in the sagittal plane with a T1-weighted spin-echo sequence of 476/15 (repetition time/echo time), with 5- and 3-mm sections for the transverse and sagittal plane, respectively. The corresponding contrast-enhanced axial and coronal T1 spin-echo sequence was obtained in 3 patients following intravenous injection of gadopentetate dimeglumine $(0.1 \mathrm{mmol} / \mathrm{kg}$; Magnevist; Bayer Schering Pharma AG, Berlin, Germany). In addition to conventional sequences, diffusion-weighted imaging (DWI) was performed with the echo planar imaging sequence with a repetition time/echo time of 5,000-6,000/90-100 ms, 5-mm contiguous sections, a field-of-view of 220x220 to 240x240 mm and a matrix size of $128 \times 128$ to $192 \times 192$. Diffusion was measured in the 6 orthogonal directions with two b-values ( 0 and $\left.1,000 \mathrm{sec} / \mathrm{mm}^{2}\right)$. The images were retrospectively reviewed by 3 neuroradiologists who were blinded to the histopathological findings.

Histopathological and immunohistochemical analysis. In all 7 cases, tissue blocks were retrieved for histopathological and immunohistochemical analysis. The resected tissues were subjected to routine processing and paraffin embedding following fixation in $10 \%$ buffered formalin. Hematoxylin and eosin staining was routinely performed. In addition, immunohistochemical assays were performed with the Dako EnVision system (including peroxidase and 3,3'-diaminobenzidine; DAKO, Carpinteria, CA, USA). All the primary antibodies used were mouse monoclonal antibodies, unless otherwise stated. These included antibodies to glial fibrillary acidic protein (1:400; 6F2, American Diagnostica GmbH, Stamford, CT, USA), synaptophysin (1:50; SY38, DakoCytomation, Glostrup Denmark), vimentin (1:100; V9, DakoCytomation), chromogranin A (1:50; DAK-A3, DakoCytomation), neurofilament protein (1:50; 2F11, DakoCytomation), S-100 (1:50; 4C4.9, rabbit polyclonal, DakoCytomation), neuron-specific enolase (1:100; BBS/NCA/I-H14, DakoCytomation), CD34 (1:100; QBEnd10; Immunotech, Marseille, France) and Ki-67 (1:50; MIB-1, DakoCytomation). All the histopathological slides were retrospectively reviewed and analyzed by a specialized neuropathologist who was blinded to the radiological findings.

\section{Results}

Clinical characteristics. The major clinical manifestations and neuroimaging findings of the 7 patients are summarized in Table I. The patients included 2 men and 5 women with a mean age of 37.6 years (range, 23-48 years) at diagnosis. The most frequent signs and symptoms included dizziness, unsteadiness of gait, headache and loss of vision, which were attributed to increased intracranial pressure or cerebellar deficits. The duration of the symptoms ranged from 4 months to 4 years, with a median duration of 20 months.

MRI analysis. All the lesions identified in the 7 patients involved the unilateral cerebellar cortex. Among these, 3 lesions were located in the right and 4 in the left cerebellar hemisphere, with involvement of the vermis in 3 patients. Hydrocephalus was observed in 4 cases and secondary tonsillar herniation was present in 4 cases. The size of the lesions ranged from 29 to $68 \mathrm{~mm}$ in greatest diameter, with a mean diameter of $50 \mathrm{~mm}$. Gross total or subtotal resections were performed in all the patients. There was no reported recurrence during a follow-up period of 7 months to 11 years. Of the 7 patients, 5 met the operational criteria established by the International Cowden Consortium. The patients developed or presented with signs of additional abnormalities associated with $\mathrm{CD}$, the most common being breast tumor $(n=4)$. All 5 patients with aLDD associated with CD were female (Table I).

On non-enhanced computed tomography (CT) of 3 patients, 1 lesion was not clearly seen and the other 2 lesions exhibited non-specific hypoattenuation. No calcification was observed. By contrast, MRI depicted the mass lesions more accurately compared to CT scanning. Multiplanar MRI images revealed a 'striated' mass involving an enlarged cerebellar hemisphere, with alternating bands of hyper- and isointensity compared to 
Table I. Patient characteristics, MRI findings and follow-up data.

\begin{tabular}{|c|c|c|c|c|}
\hline No. & Age $^{\mathrm{a}}$ gender & $\begin{array}{l}\text { Presenting symptoms } \\
\text { and signs/duration }\end{array}$ & MRI findings & Associated lesions suggestive of CD \\
\hline 1 & $23 / \mathrm{M}$ & Headache, dizziness/4 months & $\begin{array}{l}\text { Typical }{ }^{\text {b }} \text { hydrocephalus; } \\
\text { L; tonsillar herniation }\end{array}$ & - \\
\hline 2 & $48 / \mathrm{F}$ & $\begin{array}{l}\text { Intermittent dizziness } / 3 \text { years; } \\
\text { unsteady gait } / 6 \text { months }\end{array}$ & $\begin{array}{l}\text { Typical }{ }^{\text {b }} \text {, hydrocephalus; } \\
\text { L; tonsillar herniation }\end{array}$ & $\begin{array}{l}\text { Tubular carcinoma of the breast; } \\
\text { multinodular goiter; leiomyoma } \\
\text { uteri; papillomatous lesions }\end{array}$ \\
\hline 3 & $44 / \mathrm{F}$ & Unsteady gait/4 years & $\begin{array}{l}\text { Typical }^{\text {b }}+\text { hydro/Chiari; } \\
\text { R; tonsillar herniation }\end{array}$ & $\begin{array}{l}\text { Ductal carcinoma in situ } \\
\text { of the breast; facial papules }\end{array}$ \\
\hline 4 & $46 / \mathrm{F}$ & $\begin{array}{l}\text { Visual disturbance } \\
\text { of the left eye/1 year }\end{array}$ & $\begin{array}{l}\text { Typical }{ }^{\mathrm{b}} ; \mathrm{R} ; \text { tonsillar } \\
\text { herniation }\end{array}$ & $\begin{array}{l}\text { Tubular adenoma of the colon; } \\
\text { mucous cell carcinoma of } \\
\text { the stomach; acral keratoses }\end{array}$ \\
\hline 5 & $32 / \mathrm{F}$ & Intermittent dizziness/2 years & $\begin{array}{l}\text { Typical }{ }^{\mathrm{b}} ; \mathrm{R} ; \text { tonsillar } \\
\text { herniation }\end{array}$ & $\begin{array}{l}\text { Tubular carcinoma of the breast; } \\
\text { uterine fibroids }\end{array}$ \\
\hline 6 & $36 / \mathrm{M}$ & $\begin{array}{l}\text { Headache, nausea, } \\
\text { vomiting/6 months }\end{array}$ & $\begin{array}{l}\text { Typical }{ }^{\text {b; }} \text { L; hydrocephalus; } \\
\text { tonsillar herniation }\end{array}$ & - \\
\hline 7 & $34 / \mathrm{F}$ & Headache, nausea/1 year & Typical' $;$ L & $\begin{array}{l}\text { Follicular thyroid cancer; fibromas } \\
\text { of the breast; uterine fibroids }\end{array}$ \\
\hline
\end{tabular}

${ }^{\mathrm{a} A g e}$ (years) at diagnosis of aLDD. 'Lesion with a typical striated appearance on MRI. aLDD, adult-onset Lhermitte-Duclos disease; MRI, magnetic resonance imaging; CD, Cowden's disease; M, male; F, female; L, left hemisphere; R, right hemisphere; hydro, hydrocephalus.

the gray matter on T2-weighted imaging (Fig. 1). The striated appearance on $\mathrm{T} 2$-weighted imaging presented with an alteration of iso- and hypointense stripes on T1-weighted imaging. A total of 4 and 3 lesions were located in the left and right cerebellar hemispheres, respectively. There were no intratumoral necrotic regions or significant peritumoral edema in any of the patients.

Our patients exhibited additional MRI abnormalities, including involvement of the vermis $(n=2)$ and variable hydrocephalus $(n=5)$, which was a frequent finding in these patients. Furthermore, tonsillar herniation was associated with variable hydrocephalus ( $n=5)$. Three lesions were evaluated by postcontrast T1-weighted MR images and exhibited no enhancement (Fig. 1D). The morphological characteristics on MRI were uniform in the 7 patients, irrespective of coexisting CD. An illustrative case of isolated aLDD (case 4) is shown in Fig. 2. On FLAIR imaging, the mass was hyperintense in 1 of the 7 cases. In addition to conventional MRI, DWI was performed in 2 patients. DWI of high $b$-value $\left(b=1,000 \mathrm{sec} / \mathrm{mm}^{2}\right)$ revealed no restricted diffusion in the lesion and the apparent diffusion coefficient (ADC) maps demonstrated increased signal intensity compared to the contralateral normal cerebellum (Fig. 3), reflecting an increased diffusion of water. Proton MR spectroscopy was performed in 1 patient. Reduced NA/Cho and NA/Cr ratios were observed compared to the controls, with peaks attributable to lactate.

Histopathological and immunohistochemical analysis. Macroscopically, a discrete region of hypertrophy was observed within the affected cerebellum. The involved cerebellar folia were distorted and enlarged on gross examination. There was no sharp demarcation of abnormal cerebellar tissue from the adjacent tissues. Histopathologically, the widened molecular layer was occupied by abnormally hypertrophic ganglion cells. Moreover, an attenuation in the number or absence of Purkinje cells, abnormal molecular layer myelination and hypertrophy of the granular cell layer were identified, with the cellular enlargement being mainly restricted to the internal granular cell layer. These findings, together with the relative preservation of the cerebellar architecture, were consistent with the diagnosis of LDD (Fig. 4A and B). Ectopic cells morphologically consistent with granular neurons were sporadically identified in the molecular layer. Variable levels of vacuolization of the white matter were observed in all 7 cases, which was mainly located in the molecular layer (Fig. 4C). Other accompanying histopathological characteristics included calcification and ectatic vessels (Fig. 5D). There were no mitoses or pleomorphism in these cell populations.

The immunohistochemical analysis revealed no proliferative activity in the lesions, as determined by staining with monoclonal antibodies to Ki-67 (MIB-1). The ganglionic component variably expressed synaptophysin (Fig. 5A) and Neu-N (Fig. 5B). Notably, relatively diffuse immunoreactivity for CD34 was commonly observed within the lesions as determined by CD34 immunohistochemical analysis (Fig. 5C).

Our systematic retrospective search revealed that aLDD associated with $\mathrm{CD}$ exhibited no distinct pathological or immunohistochemical characteristics compared to those of isolated aLDD.

\section{Discussion}

LDD is a rare, slowly enlarging, benign entity that may occur at any age, with a peak onset in later life. In this study, 6 of our 7 patients who were diagnosed with LDD were aged 23-48 years. LDD exhibits a female preponderance; in the present series, 5 of the 7 patients were women. LDD may be 

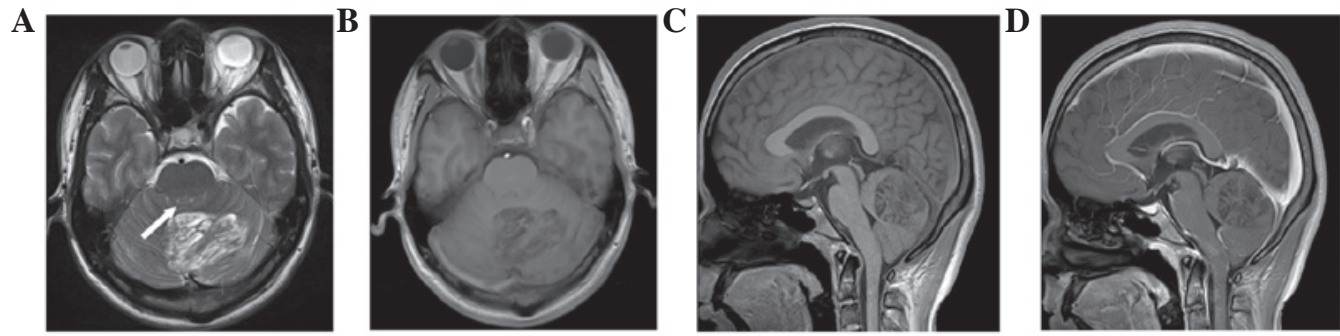

Figure 1. Typical MR images of aLDD in a 23-year-old man. (A) Axial T2- (B) T1- and (C) sagittal T1-weighted MR images of the brain display a highly characteristic non-enhancing striated appearance of aLDD. The fourth ventricle (arrow) is compressed and the vermis is involved. (D) Sagittal contrast-enhanced T1-weighted MR image reveals no enhancement following injection of the contrast agent Gd-DTPA. MR, magnetic resonance; aLDD, adult-onset Lhermitte-Duclos disease.
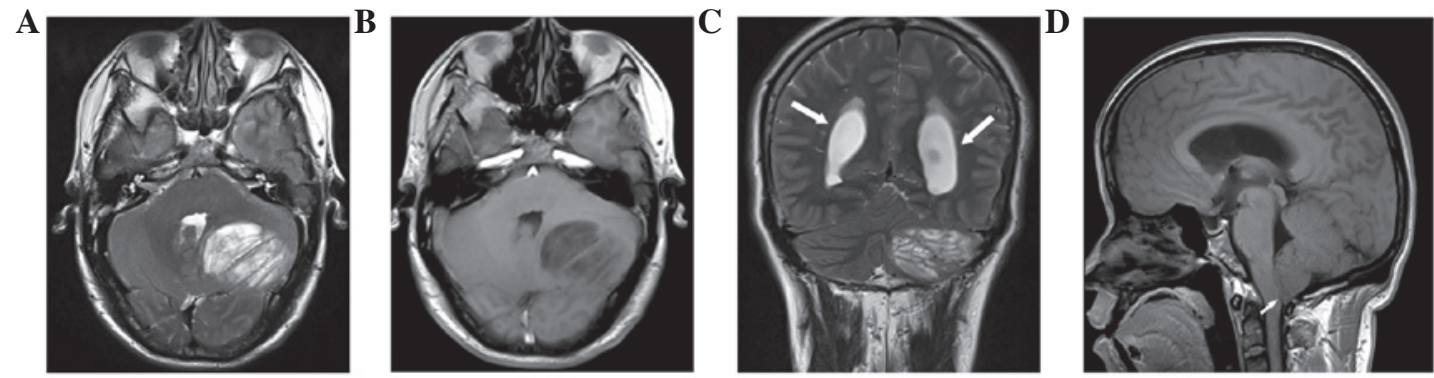

Figure 2. MRI of aLDD of the left cerebellar hemisphere in a 36-year-old man shows a characteristic striated pattern. (A) Axial T2-weighted MR image shows a predominantly hyperintense mass with alternating bands of hyper- and isointensity compared to the contralateral normal tissues. A slight mass effect is observed, with no obvious peritumoral edema. (B) On the T1-weighted MR image, the striated appearance presents with an alteration of iso- and hypointense stripes. Moderate hydrocephalus (arrows) and tonsillar herniation (arrow) are observed on (C) coronal T2- and (D) sagittal T1-weighted MR images, respectively. MRI, magnetic resonance imaging; aLDD, adult-onset Lhermitte-Duclos disease.
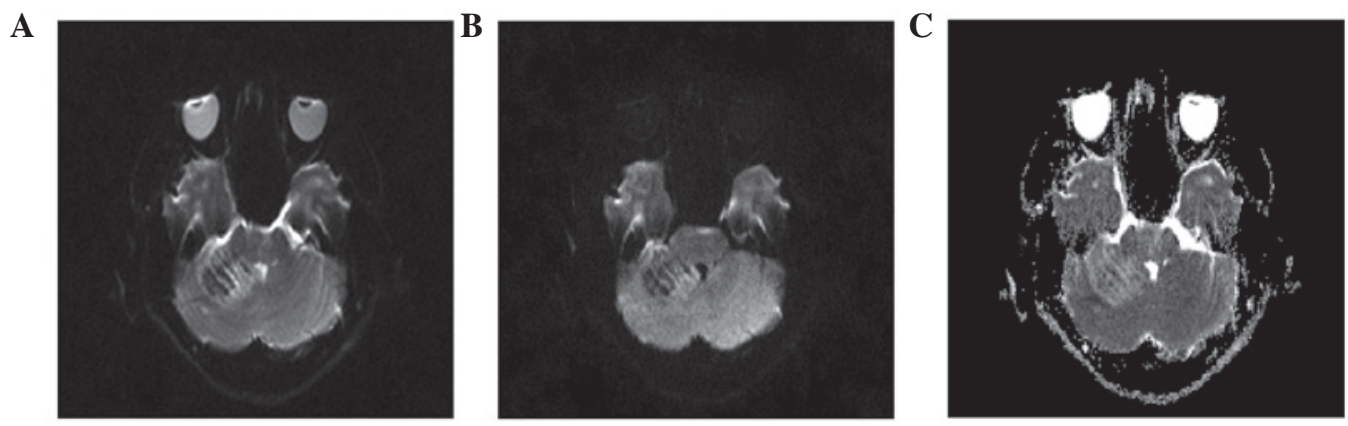

Figure 3. Diffusion-weighted imaging shows a mildly increased diffusion of water in a 46-year-old woman with adult-onset Lhermitte-Duclos disease. The lesion shows a mixed signal on axial isotropic diffusion-weighted imaging [(A) $b=0 \mathrm{sec} / \mathrm{mm}^{2}$; (B) $\left.b=1,000 \mathrm{sec} / \mathrm{mm}^{2}\right]$ and a mildly increased signal intensity compared to that of the normal brain parenchyma on the (C) corresponding apparent diffusion coefficient map, reflecting a mildly increased water diffusivity within the lesion.

manifested by a spectrum of clinical symptoms associated with the size and anatomic location of the disorder. Notably, all 7 patients suffered from long-standing symptoms, which is considered to be indicative of the slowly progressive nature of LDD.

All the cases in our study shared similar MRI characteristics, whether aLDD was sporadic or associated with CD. The appearance of LDD on MRI is pathognomonic and enables a definitive preoperative diagnosis (11-14). The hyperintense signal observed on T2-weighted images corresponds to the inner molecular and granular cell layers and the loss of central white matter within the folia (12). In addition, vacuolization of the white matter and the molecular layer may contribute to the striped pattern of the lesions on MRI (15). Enhancement in postcontrast images was not common; however, it is occasionally reported in the literature, partly due to abnormal thin-walled blood vessels (16). Our patients also exhibited MRI abnormalities such as involvement of the vermis, hydrocephalus and secondary tonsillar herniation.

Advanced MRI with novel imaging capabilities may provide insight into the underlying pathophysiology and the nature of LDD (13). Variable diffusion properties of LDD have been reported. In one of our patients, the lesion exhibited diffuse hyperintensity on the ADC maps, indicative of a benign lesion. Proton MR spectroscopy demonstrated reduced NA/Cho and $\mathrm{NA} / \mathrm{Cr}$ ratios compared to the controls, with peaks attributable to lactate $(13,17)$, which was consistent with one of our cases. The low NA/Cho and NA/Cr ratios may be attributed to the apparent lack of neuronal architecture (a hallmark of hamartoma) and the 

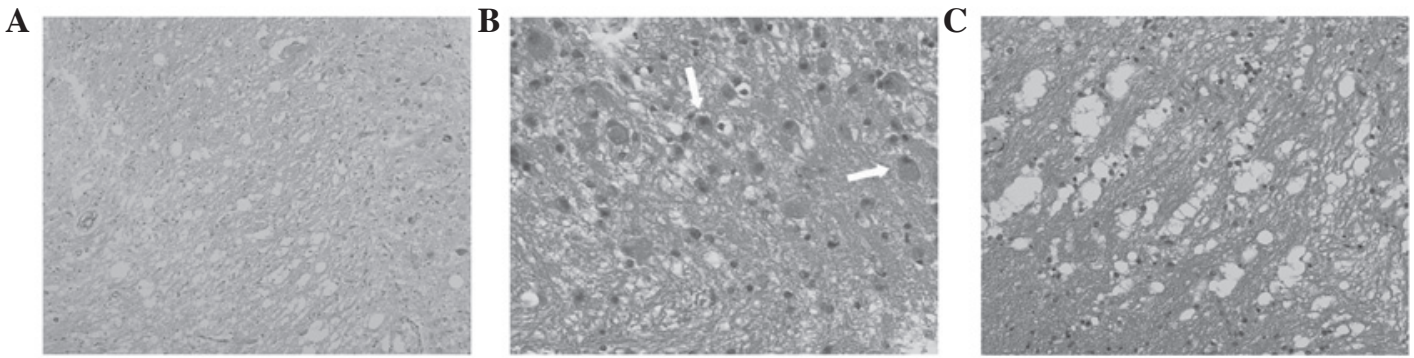

Figure 4. Histopathological examination of the resected aLDD specimens. (A) Photomicrograph [hematoxylin and eosin (H\&E) stain, original magnification, x40] shows widening of the molecular layer, absence of the Purkinje cell layer and hyperplasia of the granular cell layer. (B) Large myelinated fibers are present in the molecular layer. The large and bizarre cells are dysplastic ganglion cells with a prominent nucleus and hyperchromatic nucleoli (arrows) replacing the granular cell layer (H\&E, original magnification, $\mathrm{x} 100)$. (C) No mitosis or pleomorphism was observed in these cell populations. These characteristics are considered to be diagnostic of LDD. Vacuolization of variable levels and varying size is frequently encountered in the majority of cases and is mainly located in the white matter and in the molecular layer (H\&E, original magnification, x100). aLDD, adult-onset Lhermitte-Duclos disease.
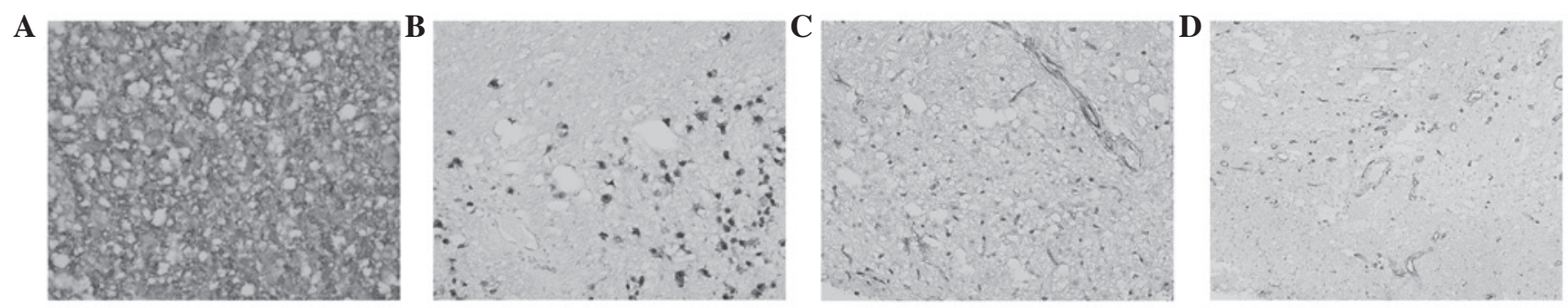

Figure 5. Immunohistochemical characteristics of adult-onset Lhermitte-Duclos disease. The enlarged cells variably expressed (A) synaptophysin and (B) Neu-N, indicative of a neuronal origin. The staining for the proliferation marker Ki-67 was negative (data not shown). (C) A relatively diffuse immunoreactivity of CD34 was observed within the lesions, as evaluated by CD34 immunohistochemistry. (D) Ectatic vessels within the lesion.

presence of embryonic neural tissue, which indicates a benign hamartoma rather than a malignant tumor (17).

LDD is pathologically characterized by regional and obvious thickening of the cerebellar folia, resulting from dysplastic replacement of cerebellar Purkinje and granular cells with hamartomatous overgrowth of hypertrophic ganglion cells. Mitotic activity and necrosis is uncommon and malignant transformation was not observed, which suggested that LDD is a hamartoma rather than a malignant tumor. Moreover, ectatic vessels are sporadically seen. Vacuolation of varying size and variable levels was also observed in the white matter, which was consistent with the findings of Abel et al (15). Following careful retrospective histopathological analysis, we observed similar histological characteristics between sporadic cases of aLDD and those with coexisting CD. Furthermore, pathological angiogenesis is commonly associated with malignant neoplasms. However, angiogenesis was frequently detected by CD34 immunohistochemistry in our series, which may be attributed to the loss of phosphatase and tensin homolog (PTEN) function on the phosphoinositol 3-kinase (PI3K)/Akt pathway and PTEN-mediated control of angiogenesis (18).

$\mathrm{CD}$ is an autosomal dominant hereditary cancer syndrome characterized by hamartomatous overgrowth of tissues of all three embryonic origins and increased risk of thyroid, breast and possibly other types of cancer $(5-7,19,20)$. In 1995, the International Cowden Consortium established a set of strict operational diagnostic criteria for CD. Furthermore, the diagnostic criteria were revised in 2000 and 2004 and are now updated annually by the US National Comprehensive Cancer Network (NCCN) $(20,21)$.
LDD occurs either sporadically or in association with CD. It was reported that aLDD is strongly associated with $\mathrm{CD}$ and the presence of a PTEN mutation $(2,5,15,20)$, as a cancer-prone disease. Based on recent data, aLDD is currently considered to be pathognomonic for $\mathrm{CD}$ (2-5), as the majority of patients with LDD either eventually fulfill the diagnostic criteria of $\mathrm{CD}$ or harbor at least one other characteristic. Zhou et al (2) performed PTEN analysis on the DNA of 18 patients with LDD; PTEN mutations were identified in all the aLDD patients $(15 / 15)$, but in none of the three control children. Germline PTEN mutations may account for the majority of the cases of LDD (20), with or without signs indicative of CD. Abel et al (15) reviewed 31 cases of LDD and analyzed the key members of the PTEN/Akt/mammalian target of rapamycin (mTOR) pathway by immunohistochemistry. Their data indicated activation of this pathway and suggested a central role for mTOR in the pathogenesis of LDD. Due to the high prevalence of PTEN mutations observed in patients with aLDD, the diagnosis of aLDD alone is sufficient for the clinical diagnosis of CD (5). A previous study reviewed 14 patients with childhood-onset LDD; however, only 3 patients were diagnosed with CD (4).

The improved awareness regarding the under-recognized association between LDD and CD, prompts a systematic search of manifestations suggestive of CD when aLDD is diagnosed. A recent literature review identified 208 LDD cases reported after 1981, of which only 53 were associated with CD (3,8,9,22-25). Notably, Robinson and Cohen (3) reported that 5 patients with LDD who were treated at their institution over a period of 40 years exhibited manifestations of $\mathrm{CD}$, including 3 previously unrecognized cases. In our 
series, 5 of the 7 cases of LDD presented evidence suggestive of $\mathrm{CD}$. Furthermore, all these 5 patients were women, suggesting a female preponderance in the incidence of LDD coexisting with $\mathrm{CD}$, which is in concordance with previous findings ( $\mathrm{n}=53$; 39 women and 14 men) $(3,8-10)$.

It is of great significance and clinical relevance to acknowledge the association between LDD and CD, although further data collection is required at the molecular level to elucidate the nature of this association. Individuals with $\mathrm{CD}$ are at an increased risk of developing benign and malignant tumors of the breast, thyroid gland and endometrium $(5,6)$. More recently, patients with PTEN mutations were also reported to exhibit an increased lifetime risk of colorectal and kidney cancer, melanoma and gastrointestinal polyps $(7,26)$.

The coexistence of LDD and CD highlights several issues. First, the high frequency of aLDD occurrence in the context of $\mathrm{CD}$ warrants the exclusion of this latter disorder in all cases of aLDD, or rather, the diagnosis of either disorder in a patient should prompt a thorough search for the other. Moreover, individuals diagnosed with aLDD coexisting with $C D$ or with germline PTEN mutations require long-term follow-up in order to preclude malignancies associated with this condition. Second, molecular evaluation, genetic counseling and gene-informed risk assessment of patients with aLDD should become evidence-based and warranted. Third, for patients with aLDD associated with $\mathrm{CD}$, particulary women, active cancer surveillance and preventive care is required following the guidelines set by the $\mathrm{NCCN}(20,27)$.

Gross total resection is currently the treatment of choice for aLDD, with a limited range of alternative treatment options. Concomitant with a better understanding of the disease-causing gene mutations, the biology of PTEN and the $\mathrm{PI} 3 \mathrm{~K} / \mathrm{Akt} / \mathrm{mTOR}$ pathway, further optimization of personal management of this entity with etiology-based molecular intervention may be anticipated.

In conclusion, all the patients included in this series shared similar neuroimaging characteristics that are highly pathognomonic of LDD. aLDD is strongly associated with CD and the MRI diagnosis of aLDD may serve as a predictor of increased risk of malignancies and entail active cancer surveillance and preventive care of associated neoplasms. Increasing knowledge regarding the nature of the association between aLDD and $\mathrm{CD}$, its underlying molecular pathogenesis and the affected signaling pathways, may enable a simplified classification and individualized management of such patients in the future.

\section{References}

1. Lhermitte J and Duclos P: Diffuse cerebellar cortex ganglioneuromas. Bull Assoc Fr Etude Cancer 9: 99-107, 1920 (In French).

2. Zhou XP, Marsh DJ, Morrison CD, et al: Germline inactivation of PTEN and dysregulation of the phosphoinositol-3-kinase/Akt pathway cause human Lhermitte-Duclos disease in adults. Am J Hum Genet 73: 1191-1198, 2003.

3. Robinson S and Cohen AR: Cowden disease and Lhermitte-Duclos disease: characterization of a new phakomatosis. Neurosurgery 46: 371-383, 2000.
4. Robinson S and Cohen AR: Cowden disease and Lhermitte-Duclos disease: an update. Case report and review of the literature. Neurosurg Focus 20: E6, 2006.

5. Hobert JA and Eng C: PTEN hamartoma tumor syndrome: an overview. Genet Med 11: 687-694, 2009.

6. Eng C: Mendelian genetics of rare - and not so rare - cancers. Ann NY Acad Sci 1214: 70-82, 2010.

7. Heald B, Mester J, Rybicki LA, et al: Frequent gastrointestinal polyps and colorectal adenocarcinomas in a prospective series of PTEN mutation carriers. Gastroenterology 139: 1927-1933, 2010.

8. Derrey S, Proust F, Debono B, et al: Association between Cowden syndrome and Lhermitte-Duclos disease: report of two cases and review of the literature. Surg Neurol 61: 447-454, 2004.

9. Nayil K, Wani M, Ramzan A, et al: Lhermitte-Duclos disease with syrinx: case report and literature review. Turk Neurosurg 21: 651-654,2011.

10. Calabria F, Grillea G, Zinzi M, et al: Lhermitte-Duclos disease presenting with positron emission tomography-magnetic resonance fusion imaging: a case report. J Med Case Rep. doi: 10.1186/1752-1947-6-76.

11. Meltzer CC, Smirniotopoulos JG and Jones RV: The striated cerebellum: an MR imaging sign in Lhermitte-Duclos disease (dysplastic gangliocytoma). Radiology 194: 699-703, 1995.

12. Kulkantrakorn K, Awwad EE, Levy B, et al: MRI in Lhermitte-Duclos disease. Neurology 48: 725-731, 1997.

13. Thomas B, Krishnamoorthy T, Radhakrishnan VV and Kesavadas C: Advanced MR imaging in Lhermitte-Duclos disease: moving closer to pathology and pathophysiology. Neuroradiology 49: 733-738, 2007.

14. Shinagare AB, Patil NK and Sorte SZ: Case 144: Dysplastic cerebellar gangliocytoma (Lhermitte-Duclos disease). Radiology 251: 298-303, 2009.

15. Abel TW, Baker SJ, Fraser MM, et al: Lhermitte-Duclos disease: a report of 31 cases with immunohistochemical analysis of the PTEN/AKT/mTOR pathway. J Neuropathol Exp Neurol 64: 341-349, 2005.

16. Spaargaren L, Cras P, Bomhof MA, et al: Contrast enhancement in Lhermitte-Duclos disease of the cerebellum: correlation of imaging with neuropathology in two cases. Neuroradiology 45: 381-385, 2003.

17. Nagaraja S, Powell T, Griffiths PD and Wilkinson ID: MR imaging and spectroscopy in Lhermitte-Duclos disease. Neuroradiology 46: 355-358, 2004.

18. Wen S, Stolarov J, Myers MP, et al: PTEN controls tumor-induced angiogenesis. Proc Natl Acad Sci USA 98: 4622-4627, 2001.

19. Marsh DJ, Coulon V, Lunetta KL, et al: Mutation spectrum and genotype-phenotype analyses in Cowden disease and Bannayan-Zonana syndrome, two hamartoma syndromes with germline PTEN mutation. Hum Mol Genet 7: 507-515, 1998.

20. Pilarski R and Eng C: Will the real Cowden syndrome please stand up (again)? Expanding mutational and clinical spectra of the PTEN hamartoma tumour syndrome. J Med Genet 41: 323-326, 2004

21. Blumenthal GM and Dennis PA: PTEN hamartoma tumor syndromes. Eur J Hum Genet 16: 1289-1300, 2008.

22. Peltier J, Lok C, Fichten A, et al: Lhermitte-Duclos disease and Cowden's syndrome. Report of two cases.Neurochirurgie 52: 407-414, 2006

23. Tan TC and Ho LC: Lhermitte-Duclos disease associated with Cowden syndrome.J Clin Neurosci 14: 801-805, 2007.

24. Zhou L, Luo L, Hui X, et al: Three adolescents with Lhermitte-Duclos disease.J Clin Neurosci: Nov 14, 2008 (Epub ahead of print). doi: 10.1016/j.jocn.2008.02.023.

25. Govindan A, Premkumar S and Alapatt JP: Lhermitte-Duclos disease (dysplastic gangliocytoma of the cerebellum) as a component of Cowden syndrome. Indian J Pathol Microbiol. doi: 10.4103/0377-4929.94875.

26. Tan MH, Mester JL, Ngeow J, et al: Lifetime cancer risks in individuals with germline PTEN mutations. Clin Cancer Res 18: 400-407, 2012

27. National Comprehensive Cancer Network: Genetic/Familial High-Risk Assessment: Breast and Ovarian. http://www.ncen. org/professionals/physician_gls/pdf/genetics_screening.pdf. Accessed July 29, 2011. 